\title{
A study of the efficiency of the class of $W$-states as a quantum channel
}

\author{
Satyabrata Adhikari; Sunandan Gangopadhyay \\ S.N. Bose National Centre for Basic Sciences, Salt Lake, Kolkata 700 098, India
}

November 26, 2018

\begin{abstract}
Recently, a new class of $W$-states has been defined by Agarwal and Pati 23] and it has been shown that they can be used as a quantum channel for teleportation and superdense coding. In this work, we identify those three-qubit states from the set of the new class of $W$-states which are most efficient or suitable for quantum teleportation. We show that with some probability $\left|W_{1}\right\rangle=\frac{1}{2}(|100\rangle+|010\rangle+\sqrt{2}|001\rangle)$ is best suited for teleportation channel in the sense that it does not depend on the input state.
\end{abstract}

PACS Numbers: 03.67.-a, 03.65.Bz,42.50.Dv

Quantum teleportation [1] involves the transmission of an arbitrary qubit from one party to another distant party by sending two classical bits. However they must initially share one entangled state. It can also be combined with other operations to construct advanced quantum circuits useful for information processing [2]. The first experimental demonstration of quantum teleportation was reported by Bouwmeester et.al. 3]. Quantum teleportation is also possible in systems corresponding to infinite dimensional Hilbert spaces [5, 6, 7, 8, 9, 10, 11].

The teleportation of quantum entanglement is also known as entanglement swapping [29, 30, 2, 4]. It enables two parties (having no common past) to share quantum entanglement. This protocol may be useful in making nonlocal correlations over long distances. Therefore it can play a significant role in quantum communication.

Quantum entanglement [12, the heart of quantum information theory, plays a crucial role in computational and communicational purposes. Therefore as a valuable resource in quantum information processing, quantum entanglement has been widely used in quantum cryptography [13, 14, quantum superdense coding [15], quantum teleportation [1, remote state preparation of special qubits and arbitrary quantum states [16, 17], and teleportation of an unitary operator [18]. Along with Einstein-Podolsky-Rosen (EPR)

\footnotetext{
${ }^{*}$ Corresponding Author: Satyabrata Adhikari, e-mail:satyabrata@bose.res.in
} 
state and Greenberger-Horne-Zeilinger (GHZ) state, there exists other entangled states such as $W$-class states and zero sum amplitude (ZSA) states [19] which have substantial importance in quantum information theory. Tripartite ZSA state may be related to $W$-class state under stochastic LOCC [20].

In 28, it was shown that an unknown qubit can be teleported using a three particle GHZ state. Joo et.al. 21] proposed quantum key distribution (QKD) and partial quantum secret sharing protocols based on $W$-states of three qubits. Further, V.N. Gorbachev et.al. [22] showed that in contrast to $|W\rangle=\frac{1}{\sqrt{3}}(|100\rangle+|010\rangle+|001\rangle)$, $|\tilde{W}\rangle=\frac{1}{2}(\sqrt{2}|100\rangle+|010\rangle+\mid 001)$ can be used as a quantum channel for teleportation of an entangled state and superdense coding. Recently, Agarwal and Pati [23] defined a large class of $W$-states that can also be used as an entanglement resource for teleportation protocol and superdense coding. Therefore, keeping in mind the importance of $W$-states, there have been various proposals to prepare these states in the literature [24, 25, 26].

In this work, we estimate the efficiency of the quantum channel $\left|W_{n}\right\rangle$ defined in [23]. To do this we proceed in the following way. First we consider a two-qubit entangled state (pure or mixed) in Alice's place. Alice then send a qubit from her entangled state to Bob using the special class of three-qubit quantum channel $\left(\left|W_{n}\right\rangle\right)$ shared by them. After the completion of the protocol, if it turns out that the amount of entanglement in Alice's two-qubit state is retained in the final two-qubit state shared by both Alice and Bob, then we can say that the efficiency of the teleportation channel is unity. If the amount of entanglement decreases after completing the teleportation protocol then the efficiency of the channel is less than unity. To quantify the above statement, we compute the concurrence [27] which gives a measure of entanglement. Hence, as it goes towards zero, one can say that the efficiency of the channel decreases.

Let us consider an arbitrary two qubit state

$$
|\psi\rangle_{12}=\alpha|00\rangle+\beta|11\rangle \quad, \quad \alpha^{2}+\beta^{2}=1 .
$$

For simplicity we assume that $\alpha$ and $\beta$ are real. As we have mentioned earlier, the above two qubit entangled state is in Alice's place, i.e. Alice holds both the particles. The special class of $W$-state is given by [23]

$$
\left|W_{n}\right\rangle_{345}=f(n)(|100\rangle+\sqrt{n}|010\rangle+\sqrt{n+1}|001\rangle)
$$

where, $n$ is a positive real number and $f(n)$ is the normalization constant given by

$$
f(n)=\frac{1}{\sqrt{(2+2 n)}} .
$$

The above three-qubit entangled state serve as a quantum channel and it is also shared by Alice and Bob. Let us assume that particle-3 is in Alice's possession and the remaining two particles (i.e. particles 4 and 5) are with Bob. Our task is to see the efficiency

\footnotetext{
${ }^{1}$ Originally there are two phase factors in $\left|W_{n}\right\rangle$ but for simplicity we have ignored them here.
} 
of this quantum channel in the teleportation protocol.

The combined system of five particles can be written as a tensor product of $|\psi\rangle_{12}$ and $\left|W_{n}\right\rangle_{345}$ :

$$
\begin{aligned}
|\chi\rangle_{12345} & =|\psi\rangle_{12} \otimes\left|W_{n}\right\rangle_{345} \\
& =\frac{f(n)}{\sqrt{2}}\left[\left|\Phi^{+}\right\rangle_{23}\left(\sqrt{n} \alpha|010\rangle_{145}+\sqrt{n+1} \alpha|001\rangle_{145}+\beta|100\rangle_{145}\right)\right. \\
& +\left|\Phi^{-}\right\rangle_{23}\left(\sqrt{n} \alpha|010\rangle_{145}+\sqrt{n+1} \alpha|001\rangle_{145}-\beta|100\rangle_{145}\right) \\
& +\left|\Psi^{+}\right\rangle_{23}\left(\alpha|000\rangle_{145}+\sqrt{n} \beta|110\rangle_{145}+\sqrt{n+1} \beta|101\rangle_{145}\right) \\
& +\left|\Psi^{-}\right\rangle_{23}\left(\alpha|000\rangle_{145}-\sqrt{n} \beta|110\rangle_{145}-\sqrt{n+1} \beta|101\rangle_{145}\right]
\end{aligned}
$$

Thereafter, Alice performs the measurement on the qubits 2 and 3 in the Bell-basis $\left\{\left|\Phi^{+}\right\rangle,\left|\Phi^{-}\right\rangle,\left|\Psi^{+}\right\rangle,\left|\Psi^{-}\right\rangle\right\}$. If the measurement outcome is $\left|\Phi^{ \pm}\right\rangle_{23}$, then it indicates that the five-qubit state $|\chi\rangle_{12345}$ collapsed to the three-qubit state $\left(\sqrt{n} \alpha|010\rangle_{145}+\right.$ $\left.\sqrt{n+1} \alpha|001\rangle_{145} \pm \beta|100\rangle_{145}\right)$. Alice then sends the measurement result to her partner Bob by spending two classical bits. After receiving the classical message from Alice, Bob performs a von-Neumann measurement on the qubit-5 in the computational basis $\{|0\rangle,|1\rangle\}$. As a result of this one-qubit measurement, the three-qubit state reduces to two-qubit state. Without any loss of generality, let us assume that von-Neumann measurement outcome is $|0\rangle_{5}$. Then the reduced two-qubit state is of the following form

$$
|\xi\rangle_{14}=N(\sqrt{n} \alpha|01\rangle \pm \beta|10\rangle)
$$

where, $N=\frac{1}{\sqrt{n \alpha^{2}+\beta^{2}}}$.

Now our task is to calculate the entanglement between the particles 1 and 4 . This calculation of the entanglement gives us insight about the efficiency of the quantum channel $\left|W_{n}\right\rangle$ in the teleportation protocol.

To do this, we recall that the concurrence of a pure state of two qubits is defined as [27]

$$
C(|\eta\rangle)=|\langle\eta \mid \tilde{\eta}\rangle|
$$

where, $|\tilde{\eta}\rangle$ denotes the spin flip of two-qubit pure state and is defined by

$$
|\tilde{\eta}\rangle=\left(\sigma_{y} \otimes \sigma_{y}\right)\left|\eta^{*}\right\rangle
$$

with $\left|\eta^{*}\right\rangle$ being the complex conjugate of $|\eta\rangle$ when it is expressed in the computational basis $\{|0\rangle,|1\rangle\}$ and

$$
\sigma_{y} \otimes \sigma_{y}=|11\rangle\langle 00|-| 01\rangle\langle 10|-| 10\rangle\langle 01|+| 00\rangle\langle 11| .
$$

With the above definitions at our disposal, we compute the concurrence for the state $|\xi\rangle_{14}$ (5) and get

$$
C\left(|\xi\rangle_{14}\right)=\frac{2 \alpha \sqrt{n\left(1-\alpha^{2}\right)}}{(n-1) \alpha^{2}+1}=C\left(|\psi\rangle_{12}\right)\left[\frac{\sqrt{n}}{(n-1) \alpha^{2}+1}\right]
$$


where, $C\left(|\psi\rangle_{12}\right)=2 \alpha \sqrt{1-\alpha^{2}}$.

Note that the concurrence $C\left(|\xi\rangle_{14}\right)$ depends on the input entangled state parameter $\alpha$ and $n$. For any fixed real positive number $n$, it can be easily observed that as $\alpha^{2}$ varies from 0 to 1, the concurrence first increases (from $C\left(|\xi\rangle_{14}\right)=0$ ), attains the maximum value $\left(C\left(|\xi\rangle_{14}\right)=1\right)$ and again decreases to zero.

Next we make the following interesting observations for the case when Alice gets the Bell-state measurement result $\left|\Phi^{ \pm}\right\rangle_{23}$ and Bob gets the von-Neumann measurement result $|0\rangle$ :

1. For $n=1$, the final concurrence $C\left(|\xi\rangle_{14}\right)$ (i.e. concurrence after completing the protocol) is exactly equal to the initial concurrence $C\left(|\psi\rangle_{12}\right)$. This means $\left|W_{1}\right\rangle$ serve as the best teleportation channel for any arbitrary input state (1). For $n \neq 1$, one can once again have $C\left(|\xi\rangle_{14}\right)=C\left(|\psi\rangle_{12}\right)$ for some specific states with parameter $\alpha$ taking the form $\alpha^{2}=\frac{\sqrt{n}-1}{n-1}$.

2. There also exist other three-qubit quantum channels $\left|W_{n}\right\rangle$ (which can be used for teleportation) for which $C\left(|\xi\rangle_{14}\right)<C\left(|\psi\rangle_{12}\right)$ and hence are not as much efficient as the state independent quantum channel $\left|W_{1}\right\rangle$ or the state dependent quantum channels $\left|W_{n}\right\rangle(n \neq 1)$ with $\alpha^{2}=\frac{\sqrt{n}-1}{n-1}$. We now classify those states as follows :

(i) For $0<n<1$, the range for $\alpha^{2}$ is given by $0<\alpha^{2}<\frac{\sqrt{n}-1}{n-1}$.

(ii)For $n>1$, the range for $\alpha^{2}$ is given by $\frac{\sqrt{n}-1}{n-1}<\alpha^{2}<1$.

Apart from the above observations, we also note that whatever be the Bell-state measurement result (obtained by Alice on qubits 2 and 3), the final concurrence (i.e. the entanglement of the final state) vanishes if Bob gets the von-Neumann measurement result $|1\rangle_{5}$. This indicates that the special class of $W$-states does not act as a good teleportation channel at all. Furthermore, the concurrence of the final state (when the Bell-state measurement result of Alice is $\left|\Psi^{ \pm}\right\rangle_{23}$ followed by Bob's von-Neumann measurement result $|0\rangle_{5}$ ) is given by :

$$
C\left(|\xi\rangle_{14}\right)=\frac{2 \beta \sqrt{n\left(1-\beta^{2}\right)}}{(n-1) \beta^{2}+1}=C\left(|\psi\rangle_{12}\right)\left[\frac{\sqrt{n}}{(n-1) \beta^{2}+1}\right] .
$$

Next we extend our idea to the case of mixed state. We want to investigate the performance of $\left|W_{n}\right\rangle$ as a teleportation channel when teleporting a single-qubit mixed state. In particular, we start with the family of Werner state which is of the form

$$
\begin{gathered}
\rho_{12}=p\left|\Phi^{+}\right\rangle\left\langle\Phi^{+}\right|+\frac{1-p}{4} I \\
=\left(\begin{array}{cccc}
\frac{(1+p)}{4} & 0 & 0 & \frac{p}{2} \\
0 & \frac{(1-p)}{4} & 0 & 0 \\
0 & 0 & \frac{(1-p)}{4} & 0 \\
\frac{p}{2} & 0 & 0 & \frac{(1+p)}{4}
\end{array}\right) .
\end{gathered}
$$

Note that the concurrence of the above state is $C\left(\rho_{12}\right)=(3 p-1) / 2$. Now considering the above state (11) as an input state and then teleporting a qubit from it using the same 
procedure discussed for pure state, we measure the efficiency of the quantum channel $\left|W_{n}\right\rangle$ by computing the concurrence. Based on the two-particle followed by one-particle measurement results, we discuss the following two cases below:

Case I: If the Bell-state measurement outcome is $\left|\Phi^{ \pm}\right\rangle_{23}$ and the von-Neumann measurement result is $|0\rangle_{5}$, then after completion of the teleportation protocol, the two-qubit state will read

$$
\rho_{14}=\left(\begin{array}{cccc}
\frac{(1-p)}{8} & 0 & 0 & 0 \\
0 & \frac{n(1+p)}{8} & \frac{ \pm \sqrt{n} p}{4} & 0 \\
0 & \frac{ \pm \sqrt{n} p}{4} & \frac{n(1+p)}{8} & 0 \\
0 & 0 & 0 & \frac{n(1-p)}{8}
\end{array}\right) .
$$

The concurrence for the above state described by the density matrix $\rho_{14}$ is therefore given by

$$
C\left(\rho_{14}\right)= \begin{cases}\frac{4 \sqrt{n}(3 p-1)}{(n+1)^{2}}=C\left(\rho_{12}\right) \frac{8 \sqrt{n}}{(n+1)^{2}}, & \text { if } p>\frac{1}{3} \\ =0, & \text { if } p \leq \frac{1}{3} .\end{cases}
$$

Now since $C\left(\rho_{14}\right) \leq C\left(\rho_{12}\right)$, hence we have the following inequality satisfied by $n$

$$
n^{4}+4 n^{3}+6 n^{2}-60 n+1 \geq 0 .
$$

The above relation explicitly gives us information about those three-qubit quantum channels $\left|W_{n}\right\rangle$ which can be used as a teleportation channel for family of Werner states with parameter $p>\frac{1}{3}$. If the inequality (14) becomes an equality then it provides us information about the quantum channel with unit efficiency. If $p \leq \frac{1}{3}$, then it can be easily checked that the concurrence vanishes and hence the $\left|W_{n}\right\rangle$ quantum channel fails to teleport.

Case II: If the Bell-state measurement outcome is $\left|\Psi^{ \pm}\right\rangle_{23}$ and the von-Neumann measurement result is $|0\rangle_{5}$, then after completion of the teleportation protocol, the two-qubit state will read

$$
\rho_{14}^{\prime}=\left(\begin{array}{cccc}
\frac{(1+p)}{8} & 0 & 0 & \frac{ \pm \sqrt{n} p}{4} \\
0 & \frac{n(1-p)}{8} & 0 & 0 \\
0 & 0 & \frac{(1-p)}{8} & 0 \\
\frac{ \pm \sqrt{n} p}{4} & 0 & 0 & \frac{n(1+p)}{8}
\end{array}\right)
$$

In this case, the concurrence is exactly equal to the concurrence given in equation (13) and hence the fact about the quantum channel $\left|W_{n}\right\rangle$ remains the same as in Case I.

But unlike the above two cases, we observe that for any Bell-state measurement made by Alice on qubits 2 and 3, if the von-Neumann measurement result (made by Bob) is $|1\rangle_{5}$, then the concurrence of the resultant state vanishes and hence once again the 
$\left|W_{n}\right\rangle$-state fails to act as a teleportation channel.

To summarize, we have presented a protocol that measures the efficiency of the class of $W$-state defined in [23]. We observe that there exists two types of $\left|W_{n}\right\rangle$-state which can serve as a quantum channel for entanglement swapping. They can be classified as: (i) State independent channel and (ii) State dependent channel. $\left|W_{1}\right\rangle$ is regarded as a state independent channel because with some probability it helps in swapping the initial entanglement to the final two-qubit state for any arbitrary two-qubit input states. On the other hand, there exist quantum channels which retains the initial entanglement in the final two-qubit state only for few input two-qubit states. Hence we call these type of channels as state dependent channel. We finally extend our analysis to the mixed state also.

\section{References}

[1] C. H. Bennett et al, Phys.Rev.Lett. 70, 1895 (1993).

[2] M. A. Nielsen and I. L. Chuang, Quantum Computation and Quantum Information, (Cambridge University Press, Cambridge, 2000).

[3] D. Bouwmeester et al, Nature 390, 575 (1997).

[4] S. L. Braunstein and A. K. Pati, Quantum Computation with continuous variables, Kluwer Academic Publishers, Dordrecht, 2003).

[5] L. Vaidman, Phys. Rev. A 49, 1473 (1994).

[6] S. L. Braunstein and H. J. Kimble, Phys. Rev. Lett. 80, 869 (1998).

[7] G. Adesso and F. Illuminati, Phys. Rev. Lett. 95, 150503 (2005).

[8] F. Dell'Anno et al, Phys. Rev. A 76, 022301 (2007).

[9] P. van Loock and S.L.Braunstein, Phys. Rev. Lett. 87, 247901 (2001).

[10] S. Adhikari et al, Phys. Rev. A 77, 012337 (2008).

[11] S. Adhikari, e-print quant-ph/0802.2156.

[12] A. Einstein et al, Phys. Rev. 47, 777 (1935).

[13] C. H. Bennett and G. Brassard, Proceedings of the IEEE international Conference on Computers, System, and Signal processing, Bangalore, India, (IEEE, New York, 1984), pp. 175-179.

[14] P. W. Shor and J. Preskill, Phys. Rev. Lett. 85, 441 (2000).

[15] C. H. Bennett and S. Wiesner, Phys. Rev. Lett. 69, 2881 (1992). 
[16] A. K. Pati, Phys. Rev. A 63, 014320-1 (2001).

[17] C. H. Bennett et al, Phys. Rev. Lett. 87, 077902 (2001).

[18] S. F. Huelga et al, Phys. Rev. A 63, 042303 (2001).

[19] A. K. Pati, Pramana J. Phys. 59, 217 (2002).

[20] W. Dur et al, Phys. Rev. A 62, 062314 (2000).

[21] J. Joo et al, e-print quant-ph/0204003.

[22] V. N. Gorbachev et al, Phys. Lett. A 314, 267 (2003).

[23] P. Agarwal, and A. K. Pati, Phys. Rev. A 74, 062320 (2006).

[24] G. C. Guo, and Y. -S. Zhang, Phys. Rev. A 65, 054302 (2002).

[25] V. N. Gorbachev et al, Phys. Lett. A 310, 339 (2003).

[26] A. Biswas, and G. S. Agarwal, J. Mod. Opt. 51, 1627 (2004).

[27] W. K. Wootters, Phys. Rev. Lett. 80, 2245 (1998).

[28] A. Karlsson, and M. Bourennane, Phys. Rev. A 58, 4394 (1998).

[29] J. -W. Pan et al, Phys. Rev. Lett. 80, 3891 (1998).

[30] S. M. Tan, Phys. Rev. A 60, 2752 (1999). 